\title{
The Falicov-Kimball Model in External Magnetic Field: Orbital Effects
}

\author{
M. Wróbel, M. MierzejeWski And M.M. MAŚKA* \\ Department of Theoretical Physics, Institute of Physics, University of Silesia \\ Uniwersytecka 4, 40-007 Katowice, Poland
}

\begin{abstract}
We study thermodynamic properties of the two-dimensional (2D) Falicov-Kimball model in the presence of external magnetic field perpendicular to the lattice. The field is taken into account by the Peierls substitution in the hopping term. We show how the Hofstadter butterfly is affected by electronic correlations. In the non-interacting case the field dependent energy spectrum forms the famous Hofstadter butterfly. Our results indicate that for arbitrary nonzero interaction strength and arbitrary magnetic field there is a gap in the energy spectrum at sufficiently low temperature. The gap vanishes with increase of temperature for weak coupling, however, it persists at high temperatures if the coupling is strong enough. Numerical results have been obtained with the help of Monte Carlo technique based on a modified Metropolis algorithm.
\end{abstract}

PACS numbers:

\section{Introduction}

Strongly correlated electron systems have generated interest over the last few decades. It is widely accepted that many phenomena in condensed matter physics are connected with electronic correlations. Moreover, an experimental progress made both in confining quantum gases and in preparation of nanosystems leads to requirement of theoretical models, that allow description of correlated quantum systems in periodic potentials and under an influence of the external magnetic field. Unfortunately, taking into account of all these three factors simultaneously is intractable. While the problem of electrons in a periodic potential under influence of the external magnetic field has been investigated since the beginning of the quantum mechanics, its solutions are known only in a few cases. In particular, one may prove that in two-dimensional fermionic gases orbital effects due to the magnetic field lead the energy spectrum to form the famous Hofstadter butterfly. Incorporation of the electron correlations in such a model encounters yet unresolved problems, mostly because of lack of relevant mathematical methods.

The Hubbard model is frequently used as a starting point for studying strongly correlated systems. Originally used for a description of the metal-insulator transition, it reveals interesting solutions, describing various phenomena observed in strongly correlated systems. Despite the simplicity of the model, only a few rigorous results are known, mostly in one- or infinite-dimensional cases. Other results have been obtained with the help of approximate methods. Some attempts have been made

\footnotetext{
* corresponding author; e-mail: maciek@phys.us.edu.pl
}

to analyze the orbital effects in the Hubbard model in an external magnetic field. Some of them, based on an exact diagonalization [1], were obtained for relatively small systems and suffer from the finite size effects. The other attempts based on the mean-field approximation (MFA)[2] are questionable due to the limited applicability of the MFA in low-dimensional systems.

The lack of exact solutions for the Hubbard model and ambiguity of solutions obtained within approximate methods encouraged us to study even simpler model, i.e., the Falicov-Kimball model. It was proposed by Hubbard and Gutzwiller (for review, see, e.g., [3, 4]) as a simplification of the Hubbard model and further was redeveloped by Falicov and Kimball to study phase transitions in rare earths and transition metals [5]. The model is a limiting case of the asymmetric one-band Hubbard model where the mass of spin-down electrons goes to infinity. It describes a system consisting two kinds of fermions. One of them are itinerant particles and the others are massive and therefore localized. The only interaction in the Falicov-Kimball model is the on-site Coulomb repulsion between itinerant and localized particles. In the second quantization the model is described by the following Hamiltonian:

$$
\mathcal{H}=-t \sum_{\langle i, j\rangle} c_{i}^{\dagger} c_{j}+U \sum_{j} f_{i}^{\dagger} f_{i} c_{i}^{\dagger} c_{i},
$$

where $t$ is the hopping integral, $c_{i}^{\dagger}\left(f_{i}^{\dagger}\right)$ are the creation operators of an itinerant (localized) fermion at site $i$ and $U$ is the Coulomb interaction.

While the Falicov-Kimball model is much simpler than its predecessor, it still cannot be rigorously solved in a general case. Fortunately, a significantly larger number of exact results is known for the the Falicov-Kimball model than for the Hubbard model [6]. One of the most im- 
portant theorem, proved by Kennedy and Lieb [7], states that at low enough temperature there is a long range order for lattices of dimensionality greater than one for certain fillings and for all values of $U$. It has been shown that the ordering comes from effective correlations between states of one kind, even though the model does not contain a direct interaction of this type. There are also important approximate results for the Falicov-Kimball model (see, e.g., Ref. [8]).

It has been demonstrated that in low dimensions the Falicov-Kimball model can effectively be analyzed with the help of the classical Monte Carlo (MC) method $[9,10]$. The method is general and it is possible to apply it for different lattice geometries and for arbitrary fillings. The most important aspect is that the model allows us for investigation of much larger systems than the original Hubbard model does. This is of crucial importance in the presence of magnetic field that results in a formation of the cyclotron orbits. It is clear that diameters of the orbits should not exceed the linear system size. Consequently, in the case of the Hubbard model one can investigate only extremely high magnetic fields when the magnetic flux through the lattice cell is of the order of the flux quantum. This limitation is significantly relaxed in the case of the Falicov-Kimball model.

In the present paper we investigate a two-dimensional Falicov-Kimball model in a presence of perpendicular magnetic field. Since we analyze the spinless FalicovKimball model, the Zeeman term is absent. The effect of the Zeeman splitting was analyzed in, e.g., Refs. [11]. For $U=0$ the model reduces itself to the Azbel-Hofstadter model, the solutions of which form the famous Hofstadter butterfly. The solutions of the $U \neq 0$ case give important guidelines how the electronic correlations modify the fine structure of Hofstadter butterfly.

The outline of the paper is as follows. Section 2 briefly describes a model and a variation of the Monte Carlo method which is used to study systems with both classical and quantum degrees of freedom. In Sect. 3 we present results obtained for Falicov-Kimball model in an external, perpendicular magnetic field. Section 4 contains summary and conclusions.

\section{Model and computational method}

In our study we analyze the extended Falicov-Kimball model, described by the following Hamiltonian:

$$
\mathcal{H}=-\sum_{\langle i, j\rangle} t_{i j}(\boldsymbol{A}) c_{i}^{\dagger} c_{j}+U \sum_{j} f_{i}^{\dagger} f_{i} c_{i}^{\dagger} c_{i},
$$

where $t_{i j}(\boldsymbol{A})$ is the hopping integral depending on the magnetic field through the Peierls phase factor

$$
t_{i j}(\boldsymbol{A})=t \exp \left(\frac{e \mathrm{i}}{\hbar} \int_{R_{i}}^{R_{j}} \boldsymbol{A} \cdot \mathrm{d} \boldsymbol{r}\right),
$$

and $\boldsymbol{A}=B(-a y,(1-a) x, 0)$ is the vector potential with the parameter $a \in[0,1]$ that allows one to distinguish between the symmetric gauge $(a=1 / 2)$ and the Landau gauge $(a=0)$. In the numerical calculations we have used the symmetric gauge. The same Hamiltonian was used by Gruber et al. to analyze flux phases in the Falicov-Kimball model [12].

In our simulations we use a modified Metropolis algorithm [10]. As our system contains both itinerant fermions and localized particles, we use the grand canonical partition function in the following form:

$$
\mathcal{Z}=\sum_{\mathcal{C}} \operatorname{Tr}_{e} \mathrm{e}^{-\beta[\mathcal{H}(\mathcal{C})-\mu \hat{N}]},
$$

where $\mathcal{C}$ describes configuration of the localized states, $\beta$ is the inverse temperature and $\hat{N}$ is the operator of total number of itinerant fermions. For a given configuration $\mathcal{C}$ the Hamiltonian $\mathcal{H}(\mathcal{C})$ can be diagonalized numerically and summation over fermionic degrees of freedom gives

$$
\mathcal{Z}=\sum_{\mathcal{C}} \prod_{n}\left\{1+\mathrm{e}^{-\beta\left[E_{n}(\mathcal{C})-\mu\right]}\right\}
$$

where $E_{n}(\mathcal{C})$ is $n$-th eigenenergy of $\mathcal{H}(\mathcal{C})$. Introducing the free energy of the mobile particles

$$
\mathcal{F}_{e}(\mathcal{C})=-\frac{1}{\beta} \sum_{n} \ln \left\{1+\mathrm{e}^{-\beta\left[E_{n}(\mathcal{C})-\mu\right]}\right\},
$$

the partition function can be written in a form analogous to that used for the Ising model

$$
\mathcal{Z}=\sum_{\mathcal{C}} \mathrm{e}^{-\beta \mathcal{F}_{e}(\mathcal{C})},
$$

where the difference from the Metropolis algorithm is that we use the electronic free energy instead of the internal energy. MC simulations allow us to estimate the partition function and thermodynamic functions such as the specific heat. Position of the peak in the specific heat and in the charge density wave (CDW) susceptibility allows one to determine the temperature $T_{\mathrm{c}}$ of the transition between the ordered and disordered phases [9].

In the present paper we restrict our considerations to a special case of half-filling both for itinerant and localized states. We have performed simulations on square lattices with sizes from $10 \times 10$ to $30 \times 30$. Here, we present results for a $20 \times 20$ lattice. Although for such a system we are unable to analyze the very fine details of the energy spectrum, it is possible to obtain the general structure of the Hofstadter butterfly within a reasonable computation time.

\section{Results}

In order to the investigate thermodynamic properties of the Falicov-Kimball model with orbital effects due to magnetic field we performed simulations for different values of the interaction strength $U / t$ and for the entire range of the magnetic flux penetrating the lattice. It is convenient to use a dimensionless quantity $\alpha=\phi / \phi_{0}$, where $\phi_{0}$ is the flux quantum and $\alpha \in(0,1)$. It was shown by Kennedy and Lieb [7] that at low temperature the localized particles in the half-filled two-dimensional Falicov-Kimball model form a checkerboard pattern. At the same time the itinerant particles show the CDW ordering. To reveal the most interesting properties of the model we have performed simulations for temperatures 
below, near and above the critical temperature $T_{\mathrm{c}}$ of the transition between ordered and disordered phase. The critical temperature was determined from the peak in the specific heat versus temperature plot.

The main results are presented in Fig. 1. In the case of weak interactions, when $U \leq t$, the general structure of the Hofstadter butterfly is nicely reproduced. At temperatures below $T_{\mathrm{c}}$ the structure is similar to the energy spectrum of the noninteracting fermions, but is split near the Fermi energy by the band gap. With an increase of the temperature the gap diminishes and vanishes completely at the $T_{\mathrm{c}}$. Above $T_{\mathrm{c}}$ the structure of the field dependent density of states looks similar to the Hofstadter butterfly, but is smeared because of averaging over disordered states.

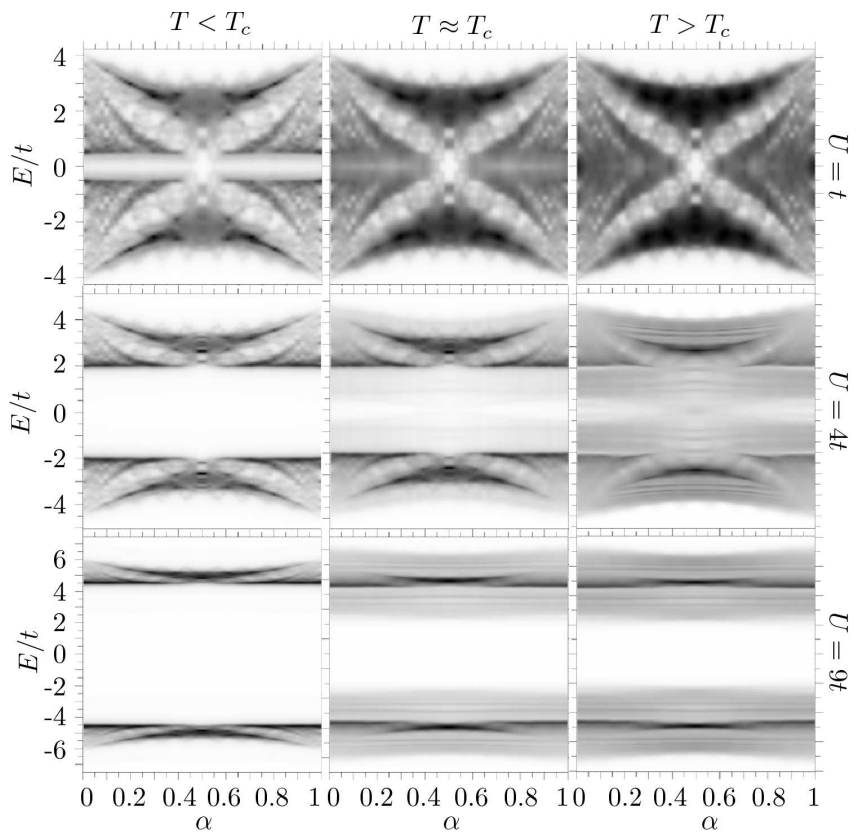

Fig. 1. Hofstadter butterflies for different $U / t$ and $k_{\mathrm{B}} T / t$. Figures were obtained for a lattice of $20 \times 20$ sites in half filling for both itinerant and localized particles. Darker color corresponds to higher value of the density of states.

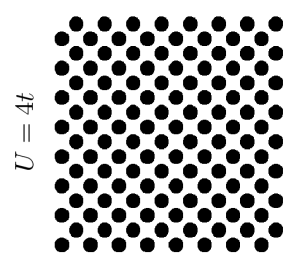

$T<T_{c}$

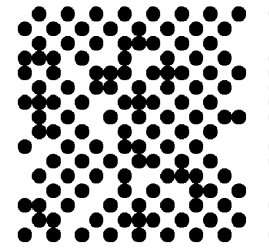

$T \approx T_{c}$

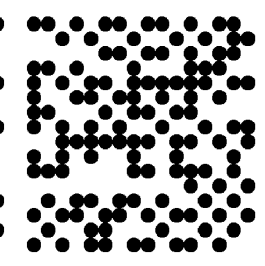

$T>T_{c}$
Fig. 2. Examples of configurations of the localized particles at different temperatures in the absence of magnetic field.

The effects of temperature on the configuration of the localized particles is illustrated in Fig. 2. The presented configurations are "snapshots" of the evolution of the system during an $\mathrm{MC}$ run. Higher interaction strengths impact the energy structure more strongly. For the intermediate interaction strengths with $U \approx 4 t$ at temperatures below $T_{\mathrm{c}}$ the energy spectrum still reproduces the Hofstadter butterfly split by the band gap. However, when the temperature increases, the obtained structure no longer resembles that for free fermions. Again at the temperature of $T_{\mathrm{c}}$ the gap vanishes, but for higher temperatures the energy spectrum seems to be weakly dependent on the magnetic field. For a strong Coulomb interaction with $U \geq 8 t$ the gap persists at arbitrary temperature and its width is not smaller than $U-8 t$. An important fact is that at zero temperature in the absence of the interaction the magnetic field switches the system between an insulating (or half-metallic in an infinite system) and a metallic phase, which results from the structure of the noninteracting Hofstadter butterfly. In the presence of the interaction this behavior is not always reproduced. At temperatures below $T_{\mathrm{c}}$ for all analyzed values of the ratio $U / t$ the sample is always in the insulating state, the same as for $U>8 t$. On the other hand, when the temperature is above $T_{\mathrm{c}}$ for weak and intermediate interaction $U \leq 2 t$ the magnetic field switches the system from metallic to insulating phase (Fig. 3).

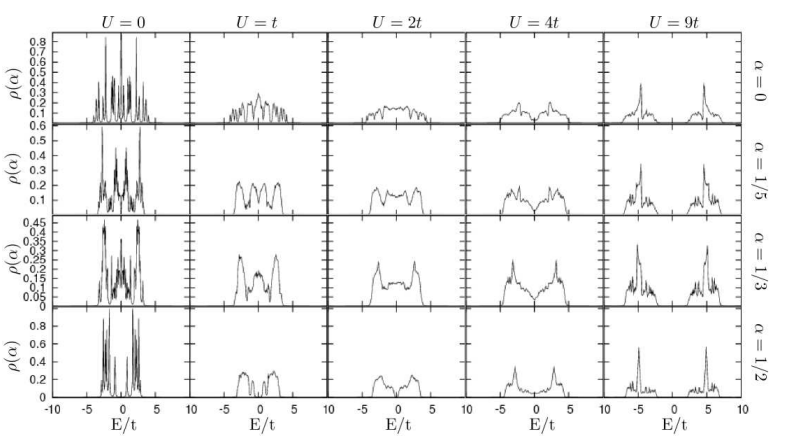

Fig. 3. Density of states obtained for various values of the magnetic flux $\alpha$ and the Coulomb repulsion $U / t$. All densities of states were obtained from simulation at temperature $T \approx 1.5 T_{\mathrm{c}}$, for a lattice of $10 \times 10$ sites with fixed boundary conditions.

\section{Conclusions and outlook}

We have shown that the numerical analysis of the Falicov-Kimball model in external magnetic field allows one to investigate the impact of the electron correlations on the Hofstadter butterfly. We investigated thermodynamics of the model with the Monte Carlo algorithm for various values of Coulomb interaction, magnetic flux and temperature. Simulations were carried out for lattices of $10 \times 10$ to $30 \times 30$ sites for half filling for both kinds of particles. For all probed values of $U$ at temperatures below the $T_{\mathrm{c}}$ states are insulating independently of magnetic field. For high values of the interaction strength $U \geq 8 t$ samples seem to be insulating at arbitrary temperature. 
An interesting result is that for weak and intermediate interaction strength the presence and magnitude of the energy gap depends on the magnetic field in irregular manner, as in the noninteracting case. We show that for all investigated values of the Coulomb repulsion there is a significant smearing of the fine fractal structure of the energy spectrum. It occurs due to a broadening of quasiparticle levels. Nevertheless, it seems that the main branches of the Hofstadter butterfly survive (though split by the energy gap) in the presence of the Coulomb interactions.

The same analysis can be repeated for the FalicovKimball model away from half filling. In this case at low temperature the localized particles form patterns which are incommensurate with the underlying lattice [13], which results in a rich structure of the density of states even without magnetic field. The fractal structure of the Hofstadter butterfly is a result of an interplay between two length scales: lattice constant and the Landau radius. In the case of the Falicov-Kimball model away from half filling there is an additional length scale, namely the period of the pattern formed by the localized particles. Therefore, one may expect the structure of the density of states versus the flux to be even richer than the Hofstadter butterfly.

Another extension of the present approach would be to take into account the spins of the itinerant and/or localized particles. It would lead to the Zeeman splitting of the energy levels, which in turn would result in drastic and nontrivial changes of the density of states at the Fermi level [14]. Therefore, in such a case the simple description of the conditions for the metal-insulator transition presented in the previous section would not be valid any more.

\section{Acknowledgments}

M.M.M. acknowledges a support by the Polish Ministry of Science and Higher Education under grant No. NN 202128736.

\section{References}

[1] K. Czajka, A. Gorczyca, M.M. Maśka, M. Mierzejewski, Phys. Rev. B 74, 125116 (2006).

[2] H. Doh, S.H. Salk, Phys. Rev. B 57, 1312 (1998).

[3] C. Gruber, D. Ueltschi, math-ph/0502041.

[4] C. Gruber, cond-mat/9811299.

[5] L.M. Falicov, J.C. Kimball, Phys. Rev. Lett. 22, 997 (1969).

[6] J.K. Freericks, V. Zlatić, Rev. Mod. Phys. 75, 1333 (2003).

[7] T. Kennedy, E. Lieb, Physica A 138, 320 (1986).

[8] J.K. Freericks, P. Miller, Phys. Rev. B 62, 10022 (2000).

[9] K. Czajka, M.M. Maśka, Physica B 359, 693 (2005).

[10] M.M. Maśka, K. Czajka, Phys. Rev. B 74, 035109 (2006).

[11] J.K. Freericks, V. Zlatić, Phys. Rev. B 58, 322 (1998); M. Žonda, cond-mat/0606242.

[12] C. Gruber, N. Marcis, A. Messager, D. Ueltschi, J. Statist. Phys. 86, 57 (1997).

[13] R. Lemański, J.K. Freericks, G. Banach, Phys. Rev. Lett. 89, 196403 (2002); K. Czajka, M.M. Maśka, Phys. Status Solidi B 244, 2427 (2007); M.M. Maśka, K. Czajka, Phys. Status Solidi B 242, 479 (2005).

[14] M.M. Maśka, Phys. Rev. B 66, 054533 (2002). 